\section{Fatores associados com a incapacidade funcional em idosos do Município de Guatambu, Santa Catarina, Brasil}

\author{
Factors associated with functional incapacity \\ among the elderly in Guatambu, \\ Santa Catarina State, Brazil
}

\author{
1 Programa de Pós-graduação \\ em Saúde Coletiva, \\ Universidade do Vale do \\ Rio dos Sinos, São Leopoldo, \\ Brasil. \\ 2 Centro Universitário \\ Leonardo da Vinci, \\ Blumenau, Brasil. \\ ${ }^{3}$ Hospital e Maternidade \\ Marieta Konder Bornhausen, \\ Itajaí, Brasil. \\ Correspondência \\ M. P. Pattussi \\ Programa de Pós-graduação \\ em Saúde Coletiva, \\ Universidade do Vale do Rio \\ dos Sinos. \\ Av. Unisinos 950, C. P. 275, \\ São Leopoldo, RS \\ 93022-000, Brasil. \\ mppattussi@unisinos.br
}

\begin{abstract}
The aim of this study was to estimate the prevalence of functional incapacity and associated factors. A cross-sectional population-based study included 352 elderly subjects ( $\geq 60$ years) in a small Brazilian town. Community-based health workers conducted structured interviews. Functional incapacity was measured by the Barthel index. Data analysis used Poisson regression to estimate crude and adjusted prevalence ratios $(P R)$ and the respective $95 \%$ confidence intervals (95\%CI). Prevalence of any kind of functional incapacity (mild, moderate, severe, or total) was 30.5\% (95\%CI: 25.6-35.2). Functional incapacity was higher $(p \leq 0.05)$ in illiterates compared to those with $>5$ years of schooling $(P R=2.21$; 95\%CI: 1.02-4.79); in people with low versus high quality of life ( $P R=1.83$; 95\%CI: 1.13-2.97); in those with poor versus good self-rated health $(P R=1.73$; 95\%CI: 1.11-2.69); and in retired individuals compared to those working regularly ( $P R=2.12 ; 95 \% C I: 1.19-3.78)$. Our results suggest that functional capacity involves interaction among multidimensional factors including selfreported health, independent daily life, and psychosocial and socioeconomic factors.
\end{abstract}

Aged; Aging; Frail Elderly; Cross-Sectional Studies
Kelly Antunes dos Santos 1,2

Ricardo Koszuoski 3

Juvenal Soares Dias-da-Costa 1

Marcos Pascoal Pattussi 1

\section{Introdução}

O processo de envelhecimento tem preocupado, especialmente nos países em desenvolvimento, quando a discussão atinge a questão da capacidade dos sistemas de saúde para acolher a crescente demanda desta faixa etária da população 1 .

Decorre daí o conceito de capacidade funcional entendido como a habilidade física e mental para manter uma vida independente e autônoma. Opostamente, a incapacidade funcional define-se pela presença de dificuldade ou mesmo pela impossibilidade no desempenho de determinadas atividades básicas da vida cotidiana 2 .

Dentre os índices que classificam pacientes idosos quanto às suas atividades básicas de vida diária, o índice de Barthel 3 é de particular importância por ser um dos instrumentos mais utilizados e possuir níveis de validade e confiabilidade aceitáveis no idioma original 4. No Brasil, não há relatos de estudos de base populacional que tenham utilizado esse índice.

O presente estudo tem como objetivo estimar a prevalência de incapacidade funcional em idosos e seus fatores associados.

\section{Métodos}

Foi realizado um estudo transversal de base populacional incluindo todas as pessoas com idade igual ou superior a 60 anos, residentes nas zonas 
urbana e rural do Município de Guatambu, Santa Catarina, Brasil. Guatambu localiza-se a $594 \mathrm{~km}$ de Florianópolis e apresentava uma população de 4.702 habitantes em 2000, destes, 371 encontravam-se na faixa etária acima de 60 anos.

A coleta de dados foi realizada por agentes comunitários de saúde e ocorreu de maio a julho de 2005. Todos os domicílios foram visitados, ocasião em que foi aplicado um questionário pré-codificado e padronizado. O controle de qualidade foi realizado em uma amostra aleatória de 10\% dos participantes.

O questionário avaliou incapacidade funcional, aspectos demográficos, sócio-econômicos, psicossociais, comportamentais e condições clínicas. Para avaliar a capacidade funcional utilizou-se a versão em português do índice de Barthel 5. O índice mede o grau de assistência exigido por um indivíduo em dez itens sobre mobilidades e cuidados pessoais. Os itens são somados atingindo-se de 0 a 100 pontos. Os pontos de corte traduzem o nível de dependência dos indivíduos. Foram encontrados poucos casos com dependência total ou grave ou moderada ( $\mathrm{n}=11$ ). Portanto, o desfecho foi categorizado como independente ou com algum tipo de dependência.

A análise dos dados foi conduzida com os programas Stata 7.0 (Stata Corp., College Station, Estados Unidos) e SPSS 10.0 (SPSS Inc., Chicago, Estados Unidos). As associações foram testadas usando-se o teste do qui-quadrado de Pearson e de associação linear. Foi utilizada regressão de Poisson para fornecer uma estimativa das razões de prevalências (RP) e seus respectivos intervalos de confiança 95\% (IC95\%). A análise multivariável foi realizada de acordo com um modelo hierarquizado. As variáveis foram ajustadas entre si no mesmo nível e para aquelas de níveis superiores que apresentavam significância estatística menor ou igual a $5 \%$.

O protocolo de pesquisa foi aprovado pelo Comitê de Bioética da Universidade do Vale do Rio dos Sinos.

\section{Resultados}

Toda a população de idosos do município foi localizada $(n=371)$. Destes, $19(5,1 \%)$ foram considerados perdas ou recusas. A maioria da população estudada era do sexo masculino, de cor branca, de idade entre 60 e 69 anos, das classes econômicas D e E e possuíam menos de dois anos de estudo (Tabela 1). Os problemas de saúde mais relatados foram: hipertensão (58\%), lombalgia (49\%), artrite/artrose (31\%) e asma/ bronquite $(22 \%)$.
A prevalência de algum tipo de incapacidade funcional (leve, moderada, grave ou total) foi igual a 30,5\% (IC95\%: 25,6-35,2). Uma maior prevalência foi encontrada em pessoas mais velhas, do sexo feminino, com menor escolaridade, de classes econômicas menos favorecidas, com qualidade de vida mais baixa, com saúde referida ruim, e com condições clínicas desfavoráveis (Tabelas 1 e 2).

O efeito das variáveis sexo, faixa etária e escolaridade foi mantido na análise multivariável. A prevalência de algum tipo de incapacidade funcional foi cerca de duas vezes maior nas pessoas analfabetas. O mesmo ocorreu com as variáveis do nível psicossocial. A incapacidade funcional das pessoas aposentadas ou em outra situação foi mais do que duas vezes a das pessoas que ainda trabalhavam. Idosos com seqüelas devido a problemas de saúde e aqueles com um maior número de morbidades possuíam uma prevalência mais elevada de incapacidade funcional (Tabela 3).

\section{Discussão}

Os resultados demonstraram que a capacidade funcional, além de estar associada com a presença de morbidades e deficiências, também é influenciada por fatores sócio-demográficos, comportamentais e psicossociais 1 .

A prevalência de incapacidade total/grave/ moderada foi de apenas 3,2\% (IC95\%: 1,3-4,9). Nesse sentido, a inclusão da categoria leve superestimou a prevalência de incapacidade funcional. Há de se considerar, que devido ao fato deste ser o único estudo de base populacional realizado no Brasil, utilizando o índice de Barthel, não são possíveis comparações nas prevalências, apenas aproximações da realidade. Por exemplo, um estudo sobre as condições de vida dos idosos realizado em São Paulo, mostrou que grande parte dos mesmos não apresentava dificuldades nas atividades básicas da vida diária e, entre aqueles que apresentaram, a maioria possuía dificuldades em uma ou duas atividades da vida diária (incapacidade leve) 6 . Além disso, o censo brasileiro de 2000 revelou que $14,5 \%$ da população brasileira possui pelo menos uma deficiência funcional relacionada. A Organização Mundial da Saúde (OMS) estima que cerca de $10 \%$ da população mundial possua deficiências e que metade destas são físico-funcionais 7 .

Neste estudo, a prevalência de apresentar algum tipo de incapacidade foi maior nas mulheres. Estudos anteriores confirmam esses achados 1,2,8,9. Essa associação provavelmente deve-se ao fato de as mulheres possuírem maior expectativa 
Distribuição da amostra e prevalência de incapacidade funcional de acordo com variáveis sócio-econômicas, demográficas, psicossociais, comportamentais e condições clínicas referidas em pessoas com 60 ou mais anos de idade. Guatambu, Santa Catarina, Brasil, 2005 (N = 352 ).

\begin{tabular}{|c|c|c|c|c|c|}
\hline \multirow[t]{2}{*}{ Variável } & \multirow[t]{2}{*}{$\mathrm{n}$} & \multirow[t]{2}{*}{$\%$} & \multicolumn{2}{|c|}{ Prevalência de incapacidade } & \multirow[t]{2}{*}{ p valor * } \\
\hline & & & $\mathrm{n}$ & $\%$ & \\
\hline \multicolumn{6}{|l|}{ Sexo } \\
\hline Masculino & 185 & 52,6 & 45 & 24,3 & $<0,001$ \\
\hline Feminino & 167 & 47,4 & 62 & 37,1 & \\
\hline Cor da pele & & & & & 0,238 \\
\hline Branca & 234 & 66,5 & 76 & 32,5 & \\
\hline Parda & 98 & 27,8 & 28 & 28,6 & \\
\hline Outra & 20 & 5,7 & 3 & 15,0 & \\
\hline Faixa etária (anos) & & & & & 0,001 \\
\hline $60-69$ & 191 & 54,3 & 45 & 23,6 & \\
\hline $70-79$ & 117 & 33,2 & 41 & 35,0 & \\
\hline 80 ou mais & 44 & 12,5 & 21 & 47,7 & \\
\hline Classe econômica ** & & & & & 0,042 \\
\hline$B$ e $C$ & 109 & 31,0 & 25 & 22,9 & \\
\hline$D e E$ & 243 & 69,0 & 82 & 37,3 & \\
\hline Escolaridade (anos) & & & & & 0,012 \\
\hline 5 ou mais & 38 & 10,8 & 6 & 15,8 & \\
\hline $3-4$ & 110 & 31,3 & 32 & 29,1 & \\
\hline $1-2$ & 76 & 21,6 & 20 & 26,3 & \\
\hline Analfabeto & 128 & 36,4 & 49 & 38,3 & \\
\hline Lazer (número de atividades) & & & & & 0,122 \\
\hline $5-6$ & 119 & 33,8 & 28 & 23,5 & \\
\hline $3-4$ & 174 & 49,4 & 60 & 34,5 & \\
\hline $0-2$ & 59 & 16,8 & 19 & 32,2 & \\
\hline Qualidade de vida *** & & & & & $<0,001$ \\
\hline Alta & 96 & 27,3 & 17 & 17,7 & \\
\hline Média & 125 & 35,5 & 33 & 26,4 & \\
\hline Baixa & 131 & 37,2 & 57 & 43,5 & \\
\hline Autopercepção de saúde & & & & & $<0,001$ \\
\hline Boa/Muito boa/Ótima & 123 & 34,9 & 21 & 17,1 & \\
\hline Regular/Ruim & 229 & 65,1 & 86 & 37,6 & \\
\hline Trabalho & & & & & $<0,001$ \\
\hline Trabalhando & 93 & 26,4 & 12 & 12,9 & \\
\hline Aposentado & 229 & 65,1 & 82 & 35,8 & \\
\hline Outro & 30 & 8,5 & 13 & 43,3 & \\
\hline Índice de massa corporal & & & & & 0,138 \\
\hline Normal & 220 & 63,0 & 74 & 33,6 & \\
\hline Sobrepeso & 91 & 26,1 & 22 & 24,2 & \\
\hline Obesidade & 38 & 10,9 & 10 & 26,3 & \\
\hline Atividade física & & & & & 0,082 \\
\hline Sim & 54 & 15,3 & 11 & 20,4 & \\
\hline Não & 298 & 84,3 & 96 & 32,2 & \\
\hline Hospitalização & & & & & 0,022 \\
\hline Nenhuma & 305 & 86,6 & 86 & 28,2 & \\
\hline Uma ou mais & 47 & 13,4 & 21 & 44,7 & \\
\hline
\end{tabular}

(continua) 
Tabela 1 (continuação)

\begin{tabular}{|c|c|c|c|c|c|}
\hline \multirow[t]{2}{*}{ Variável } & \multirow[t]{2}{*}{$\mathbf{n}$} & \multirow[t]{2}{*}{$\%$} & \multicolumn{2}{|c|}{ Prevalência de incapacidade } & \multirow[t]{2}{*}{$\mathrm{p}$ valor * } \\
\hline & & & $\mathrm{n}$ & $\%$ & \\
\hline Consultas & & & & & 0,014 \\
\hline Nenhuma & 122 & 34,7 & 27 & 22,1 & \\
\hline $1-2$ & 117 & 33,2 & 41 & 35,0 & \\
\hline $3-4$ & 67 & 19,0 & 20 & 29,9 & \\
\hline 5 ou mais & 43 & 12,2 & 19 & 44,2 & \\
\hline Morbidades & & & & & $<0,001$ \\
\hline $0-2$ & 210 & 59,7 & 43 & 20,5 & \\
\hline $3-4$ & 111 & 31,5 & 46 & 41,4 & \\
\hline 5 ou mais & 31 & 8,8 & 18 & 58,1 & \\
\hline Seqüelas & & & & & $<0,001$ \\
\hline Não & 277 & 78,7 & 75 & 27,1 & \\
\hline Sim & 75 & 21,3 & 32 & 42,7 & \\
\hline
\end{tabular}

* Qui-quadrado de Pearson e de tendência linear;

** Classificação econômica conforme Associação Brasileira de Empresas de Pesquisa 16;

*** De acordo com Power 17

Tabela 2

Razões de prevalência (RP) brutas e respectivos intervalos de confiança (IC95\%) para incapacidade funcional de acordo com variáveis sócio-econômicas, demográficas, psicossociais, comportamentais e condições clínicas referidas em pessoas com 60 ou mais anos de idade. Guatambu, Santa Catarina, Brasil, 2005 ( $N=352)$.

\begin{tabular}{|c|c|c|c|}
\hline Variável & RP & IC95\% & $\mathrm{p}$ valor * \\
\hline \multicolumn{4}{|l|}{ Sexo } \\
\hline Masculino & 1,00 & - & 0,010 \\
\hline Feminino & 1,53 & $1,10-2,11$ & \\
\hline \multicolumn{4}{|l|}{ Cor da pele } \\
\hline Branca & 1,00 & - & 0,308 \\
\hline Parda & 0,88 & $0,61-1,26$ & \\
\hline Outra & 0,46 & $0,16-1,33$ & \\
\hline \multicolumn{4}{|c|}{ Faixa etária (anos) } \\
\hline $60-69$ & 1,00 & - & 0,002 \\
\hline $70-79$ & 1,49 & $1,04-2,12$ & \\
\hline 80 ou mais & 2,02 & $1,35-3,03$ & \\
\hline \multicolumn{4}{|c|}{ Classe econômica ** } \\
\hline $\mathrm{B}$ e $\mathrm{C}$ & 1,00 & - & 0,051 \\
\hline$D$ e $E$ & 1,47 & $1,00-2,17$ & \\
\hline \multicolumn{4}{|c|}{ Escolaridade (anos) } \\
\hline 5 ou mais & 1,00 & - & 0,058 \\
\hline $3-4$ & 1,84 & $0,83-4,06$ & \\
\hline $1-2$ & 1,67 & $0,73-3,80$ & \\
\hline Analfabeto & 2,42 & $1,12-5,22$ & \\
\hline \multicolumn{4}{|c|}{ Lazer (número de atividades) } \\
\hline $5-6$ & 1,00 & - & 0,146 \\
\hline $3-4$ & 1,46 & $1,00-2,15$ & \\
\hline $0-2$ & 1,37 & $0,84-2,24$ & \\
\hline
\end{tabular}

(continua) 


\begin{tabular}{|c|c|c|c|}
\hline Variável & $\mathrm{RP}$ & IC95\% & $p$ valor * \\
\hline \multicolumn{4}{|l|}{ 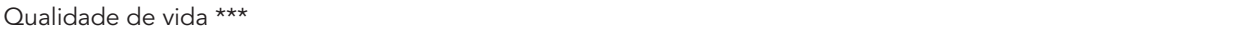 } \\
\hline Alta & 1,00 & - & $<0,001$ \\
\hline Média & 1,49 & $0,88-2,51$ & \\
\hline Baixa & 2,46 & $1,53-3,95$ & \\
\hline \multicolumn{4}{|l|}{ Autopercepção de saúde } \\
\hline Boa/Muito boa/Ótima & 1,00 & - & $<0,001$ \\
\hline Regular/Ruim & 2,20 & $1,44-3,36$ & \\
\hline \multicolumn{4}{|l|}{ Trabalho } \\
\hline Trabalhando & 1,00 & - & $<0,001$ \\
\hline Aposentado & 2,77 & $1,59-4,84$ & \\
\hline Outro & 3,36 & $1,72-6,56$ & \\
\hline \multicolumn{4}{|l|}{ Índice de massa corporal } \\
\hline Normal & 1,00 & - & 0,236 \\
\hline Sobrepeso & 0,72 & $0,48-1,08$ & \\
\hline Obesidade & 0,78 & $0,44-1,37$ & \\
\hline \multicolumn{4}{|l|}{ Atividade física } \\
\hline Sim & 1,00 & - & 0,104 \\
\hline Não & 1,58 & $0,91-2,75$ & \\
\hline \multicolumn{4}{|l|}{ Hospitalização } \\
\hline Nenhuma & 1,00 & - & 0,014 \\
\hline Uma ou mais & 1,58 & $1,01-2,28$ & \\
\hline \multicolumn{4}{|l|}{ Consultas } \\
\hline Nenhuma & 1,00 & - & 0,031 \\
\hline $1-2$ & 1,58 & $1,04-2,40$ & \\
\hline $3-4$ & 1,35 & $0,82-2,21$ & \\
\hline 5 ou mais & 1,97 & $1,24-3,20$ & \\
\hline \multicolumn{4}{|l|}{ Morbidades } \\
\hline $0-2$ & 1,00 & - & $<0,001$ \\
\hline $3-4$ & 2,02 & $1,43-2,86$ & \\
\hline 5 ou mais & 2,84 & $1,90-4,24$ & \\
\hline \multicolumn{4}{|l|}{ Seqüelas } \\
\hline Não & 1,00 & - & 0,006 \\
\hline Sim & 1,57 & $1,14-2,18$ & \\
\hline
\end{tabular}

* Teste Wald para heterogeneidade ou para tendências;

** Classificação econômica conforme Associação Brasileira de Empresas de Pesquisa 16;

*** De acordo com Power 17

de vida e, conseqüentemente, maior risco de desenvolver doenças crônicas incapacitantes.

Idosos da faixa etária mais elevada apresentaram uma maior prevalência de incapacidade funcional. Em geral, limitações funcionais são mais freqüentes em indivíduos mais idosos pela longevidade $1,7,10$.

Neste estudo, idosos com baixa escolaridade e classes menos favorecidas apresentaram maior incapacidade funcional, achado este relatado em outros estudos 1,9. Baixos níveis de educação e piores condições sócio-econômicas estão associados a maiores riscos de deficiência e morte 11. Oportunidades de aprendizado podem ajudar as pessoas a desenvolver as habilidades e confiança para se adaptar e desenvolver um processo de envelhecimento saudável. Idosos com melhores condições financeiras geralmente possuem melhor acesso à prevenção, tratamento e reabilitação.

Nesta pesquisa, os idosos que apresentaram baixa qualidade de vida e os que possuíam uma percepção ruim de sua saúde apresentaram maiores níveis de dependência funcional. Um estudo revelou que fatores que se mantiveram associados ao envelhecimento bem-sucedido incluíam apoio psicossocial, autopercepção de saúde favorável e relações com a família e amigos 12. Outros estudos têm demonstrado a associação entre incapacidade funcional e autopercepção de saúde desfavorável 1 . Na condição 
Tabela 3

Razões de prevalência (RP) ajustadas e respectivos intervalos de confiança (IC95\%) para incapacidade funcional em pessoas com 60 ou mais anos de idade. Guatambu, Santa Catarina, Brasil, 2005 (N = 352)

\begin{tabular}{|c|c|c|c|c|}
\hline Modelo & Variável & $\mathrm{RP}$ & IC95\% & $\mathrm{p}$ valor * \\
\hline \multirow[t]{19}{*}{ Bloco 1: sócio-demográfico ** } & Sexo & & & \\
\hline & Masculino & 1,00 & - & 0,029 \\
\hline & Feminino & 1,42 & $1,03-1,96$ & \\
\hline & Cor da pele & & & \\
\hline & Branca & 1,00 & - & 0,080 \\
\hline & Parda & 0,81 & $0,57-1,15$ & 0,149 \\
\hline & Outra & 0,42 & $0,16-1,10$ & 0,066 \\
\hline & Faixa etária (anos) & & & \\
\hline & $60-69$ & 1,00 & - & 0,039 \\
\hline & $70-79$ & 1,31 & $0,91-1,88$ & 0,158 \\
\hline & 80 ou mais & 1,73 & $1,16-2,59$ & 0,011 \\
\hline & 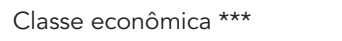 & & & \\
\hline & $\mathrm{B}$ e $\mathrm{C}$ & 1,00 & - & 0,167 \\
\hline & $D$ e $E$ & 1,32 & $0,89-1,97$ & \\
\hline & Escolaridade (anos) & & & \\
\hline & 5 ou mais & 1,00 & - & 0,136 \\
\hline & $3-4$ & 1,80 & $0,82-3,99$ & 0,144 \\
\hline & $1-2$ & 1,58 & $0,70-3,62$ & 0,275 \\
\hline & Analfabeto & 2,21 & $1,02-4,79$ & 0,045 \\
\hline \multirow[t]{11}{*}{ Bloco 2: psicossociais \# } & Lazer (número de atividades) & & & \\
\hline & $5-9$ & 1,00 & - & 0,507 \\
\hline & $3-4$ & 1,26 & $0,85-1,87$ & 0,207 \\
\hline & $0-2$ & 1,21 & $0,75-1,94$ & 0,430 \\
\hline & Qualidade de vida \#\# & & & \\
\hline & Alta & 1,00 & - & 0,010 \\
\hline & Média & 1,16 & $0,70-2,03$ & 0,506 \\
\hline & Baixa & 1,77 & $1,13-2,97$ & 0,022 \\
\hline & Autopercepção de saúde & & & \\
\hline & Boa/Muito boa/Ótima & 1,00 & - & 0,012 \\
\hline & Regular/Ruim & 1,76 & $1,13-2,72$ & \\
\hline \multirow[t]{11}{*}{ Bloco 3: comportamentais \#\#\# } & Trabalho & & & \\
\hline & Trabalhando & 1,00 & - & 0,009 \\
\hline & Aposentado & 2,12 & $1,19-3,78$ & 0,012 \\
\hline & Outro & 2,88 & $1,47-5,63$ & 0,002 \\
\hline & Índice de massa corporal & & & \\
\hline & Normal & 1,00 & - & 0,898 \\
\hline & Sobrepeso & 0,96 & $0,65-1,43$ & 0,856 \\
\hline & Obesidade & 0,87 & $0,53-1,44$ & 0,592 \\
\hline & Atividade física & & & \\
\hline & Sim & 1,00 & - & 0,198 \\
\hline & Não & 1,47 & $0,82-2,64$ & \\
\hline
\end{tabular}

(continua) 


\begin{tabular}{|c|c|c|c|c|}
\hline Modelo & Variável & $\mathrm{RP}$ & IC95\% & $\mathrm{p}$ valor * \\
\hline \multirow[t]{15}{*}{ Bloco 4: clínicas § } & Hospitalização & & & \\
\hline & Nenhuma & 1,00 & - & 0,826 \\
\hline & Uma ou mais & 1,04 & $0,70-1,55$ & \\
\hline & Consultas & & & \\
\hline & Nenhuma & 1,00 & - & 0,697 \\
\hline & $1-2$ & 1,02 & $0,65-1,60$ & 0,936 \\
\hline & $3-4$ & 0,81 & $0,48-1,37$ & 0,434 \\
\hline & 5 ou mais & 0,88 & $0,49-1,56$ & 0,665 \\
\hline & Morbidades & & & \\
\hline & $0-2$ & 1,00 & - & 0,013 \\
\hline & $3-4$ & 1,61 & $1,12-2,33$ & 0,011 \\
\hline & 5 ou mais & 1,75 & $1,17-2,65$ & 0,007 \\
\hline & Seqüelas & & & \\
\hline & Não & 1,00 & - & 0,040 \\
\hline & Sim & 1,40 & $1,02-1,92$ & \\
\hline
\end{tabular}

* Teste Wald para heterogeneidade ou para tendências;

** Ajustadas entre si;

*** Classificação econômica conforme Associação Brasileira de Empresas de Pesquisa 16;

\# Ajustadas entre si e para sexo, faixa etária e escolaridade;

\#\# De acordo com Power 17;

\#\#\# Ajustadas entre si e para sexo, faixa etária, escolaridade, qualidade de vida e autopercepção de saúde;

$\S$ Ajustadas entre si e para sexo, faixa etária, escolaridade, qualidade de vida, autopercepção de saúde e trabalho.

comportamental, os idosos que mais apresentaram algum tipo de dependência física foram os aposentados. Provavelmente exista um efeito protetor no fato de ocupar-se com o trabalho, mantendo corpo e mente ativos até idades mais avançadas 1 .

Neste estudo, os idosos que foram hospitalizados, que mais consultaram, com maior número de morbidades e aqueles que haviam sofrido algum acidente ou patologia com seqüelas apresentaram uma maior prevalência de algum tipo de dependência. Doenças crônicas não transmissíveis são mais freqüentes entre os idosos, e são justamente as mais incapacitantes 13 . A associação com variáveis clínicas como hospitalizações, consultas e morbidades tem sido relatada em vários estudos 1,2,14. A associação com seqüelas é um resultado esperado, visto que, o fato de possuir seqüela devido a alguma patologia ou acidente está intimamente relacionado à incapacidade funcional.

Capacidade funcional de indivíduos idosos surge como novo conceito em saúde, já que a presença de doenças não está relacionada à perda de sua autonomia. Envelhecimento bem-sucedido passa a ser o resultado da interação de fatores multidimensionais, os quais incluem questões relacionadas à saúde física, mental, independência na vida diária, aspectos econômicos e psicossociais 15. Com isso o foco de atenção ao idoso é ampliado e melhores estratégias de planejamento, implementação e avaliação de ações podem ser encontradas com vistas à melhoria da qualidade de vida de uma população que embora seja a que mais cresça em nosso país, ainda é uma das mais excluídas das políticas públicas. 


\section{Resumo}

O objetivo deste estudo era estimar a prevalência de incapacidade funcional e seus fatores associados. Foi realizado um estudo transversal de base populacional (censo) com 352 pessoas com idade maior ou igual 60 anos do Município de Guatambu, Santa Catarina, Brasil. Entrevistas foram realizadas pelos agentes de saúde. A incapacidade funcional foi medida usando-se o indice de Barthel. A análise dos dados utilizou regressão de Poisson. A prevalência de algum tipo de incapacidade funcional (leve, moderada, grave ou total) foi igual a 30,5\% (IC95\%: 25,6-35,2). A incapacidade funcional foi maior $(p \leq 0,05)$ nos analfabetos quando comparados aos com escolaridade mais elevada $(R P=2,21$; IC95\%: 1,02-4,79); nas pessoas com qualidade de vida baixa comparadas às com alta ( $R P=1,83$; IC95\%: 1,132,97); nas pessoas com saúde referida ruim/moderada comparadas às com saúde boa/muito boa/ótima $(R P=$ 1,73; IC95\%: 1,11-2,69); nas pessoas aposentadas comparadas às que ainda trabalhavam $(R P=2,12$; IC95\%: 1,19-3,78) bem como nas pessoas com maior número de morbidades e seqüelas associadas. O estudo sugere que a capacidade funcional depende da interação de fatores multidimensionais incluindo a saúde física, independência na vida diária, aspectos econômicos e psicossociais.

Idoso; Envelhecimento; Idoso Débil; Estudos Transversais

\section{Colaboradores}

K. A. Santos originou o estudo, conduziu a pesquisa e escreveu o manuscrito. R. Koszuoski e J. S. Dias-da-Costa contribuíram na conceituação e na escrita do manuscrito. M. P. Pattussi orientou o estudo e a análise e interpretação dos dados.

\section{Agradecimentos}

A todos que contribuíram na coleta de dados e aos idosos que participaram da pesquisa.

\section{Referências}

1. Rosa TEC, Benício MHDA, Latorre MRDO, Ramos LR. Fatores determinantes da capacidade funcional entre idosos. Rev Saúde Pública 2003; 37:40-8.

2. Ramos LR, Rosa TEC, Oliveira ZM, Medina MCG, Santos FRG. Perfil do idoso em área metropolitana na região sudeste do Brasil: resultados de inquérito domiciliar. Rev. Saúde Pública 1993; 27:87-94.

3. Mahoney FI, Barthel DW. Functional evaluation: the Barthel index. Md Med J 1965; 14:61-5.

4. Sainsbury A, Seebass G, Bansal A, Young JB. Reliability of the Barthel index when used with older people. Age Ageing 2005; 34:228-32.

5. Labronici RHDD, Cunha MCB, Oliveira ASB Gabbai AA. Esporte como fator de integração do deficiente físico na sociedade. Arq Neuropsiquiatr 2000; 58:1092-9.

6. Laurenti EL, Lebrão S. Self rated health and mortality: a review of twenty-seven community studies. J Health Soc Behav 2005; 38:21-37.

7. World Health Organization. Concept paper: world report on disability and rehabilitation. Geneva: World Health Organization; 2007.

8. Kington RS, Smith JP. Socioeconomic status and racial and ethnic differences in functional status associated with chronic diseases. Am J Public Health 1997; 87:805-10.

9. Parahyba MI, Veras R, Melzer D. Incapacidade funcional entre mulheres idosas no Brasil. Rev Saúde Pública 2005; 39:383-91.

10. Barbosa AR, Souza JMP, Lebrão ML, Laurenti R, Marucci MFN. Functional limitations of Brazilian elderly by age and gender differences: data from SABE Survey. Cad Saúde Pública 2005; 21:1177-85.

11. Organização Mundial da Saúde. Envelhecimento ativo: uma política de saúde. Brasília: Organização Pan-Americana da Saúde; 2005.

12. Moraes JFD, Souza VBA. Factors associated with the successful aging of the socially-active elderly in the metropolitan region of Porto Alegre. Rev Bras Psiquiatr 2005; 27:302-8.

13. Karsch UMS, organizador. Envelhecimento com dependência: revelando cuidadores. São Paulo: EDUC; 1998

14. Siqueira AB, Cordeiro RC, Perracini MR, Ramos, LR. Impacto funcional da internação hospitalar de pacientes idosos. Rev Saúde Pública 2004; 38: 687-94.

15. Ramos LR. Fatores determinantes do envelhecimento saudável em idosos residentes em centro urbano: Projeto Epidoso, São Paulo. Cad Saúde Pública 2003; 19:793-8.

16. Associação Brasileira de Empresas de Pesquisa. Critério de classificação econômica Brasil. http://www.abep.org/codigosguias/ABEP_CCEB. pdf (acessado em 21/Mar/2005).

17. Power M. Development of a common instrument for quality of life. In: Nosikov A, Gudex C, editors. Eurohis: developing common instruments for health survey. Amsterdam: IOS Press; 2003. p. 145-63.

Recebido em 08/Jan/2007

Versão final reapresentada em 29/Mai/2007

Aprovado em 25/Jun/2007 